\title{
Role of carbonic anhydrase IX expression in prediction of the efficacy and outcome of primary epirubicin/tamoxifen therapy for breast cancer
}

\author{
Daniele Generali ${ }^{1,2}$, Stephen B Fox ${ }^{1}$, Alfredo Berruti ${ }^{4}$, Maria P Brizzi ${ }^{4}$, \\ Leticia Campo ${ }^{1}$, Simone Bonardi ${ }^{2}$, Simon M Wigfield ${ }^{1}$, Paolo Bruzzi ${ }^{5}$, \\ Alessandra Bersiga ${ }^{3}$, Giovanni Allevi ${ }^{2}$, Manuela Milani ${ }^{2}$, Sergio Aguggini ${ }^{2}$, \\ Luigi Dogliotti ${ }^{4}$, Alberto Bottini ${ }^{2}$ and Adrian L Harris ${ }^{1}$
}

\footnotetext{
${ }^{1}$ Weatherall Molecular Oncology Laboratories, Institute of Molecular Medicine, John Radcliffe Hospital, University of Oxford, Oxford OX3 9DS, UK

${ }^{2}$ Unità di Patologia Mammaria - Breast Cancer Unit, Azienda Istituti Ospitalieri di Cremona, Viale Concordia 1, 26100 Cremona, Italy

${ }^{3}$ Anatomia Patologica Azienda Istituti Ospitalieri di Cremona, Viale Concordia 1, 26100 Cremona, Italy

${ }^{4}$ Oncologia Medica, Dipartimento di Scienze Cliniche e Biologiche, Università di Torino Azienda Ospedaliera San Luigi di Orbassano, Regione Gonzole 10, 10043 Orbassano, Italy

${ }^{5}$ Istituto Nazionale per la Ricerca sul Cancro, Largo R. Benzi 10, 16132 Genova, Italy

(Requests for offprints should be addressed to A L Harris; Email: aharris.lab @ cancer.org.uk)
}

\begin{abstract}
The purpose of this study is to investigate the role of carbonic anhydrase IX (CAIX) expression in predicting the response to epirubicin and disease-free survival (DFS) in breast cancer patients enrolled in a single institution trial of primary anthracycline and tamoxifen therapy. CAIX expression was assessed in 183 patients with T2-4 N0-1 breast cancer enrolled in a randomized trial comparing four cycles of single agent epirubicin versus epirubicin + tamoxifen as primary systemic treatment. All patients received postoperatively four cycles of the four weekly i.v. cyclophosphamide, methotrexate, 5-fluorouracil regimen. Patients with estrogen receptor (ER)positive primary tumors received 5 years of adjuvant tamoxifen. Pretreatment, p53 $(P=0.007)$, C-erbB2 $(P<0.01)$, and Ki67 $(P=0.02)$ were directly associated with CAIX expression, while bcl2 $(P<0.000)$ and ER $(P=0.000)$ and progesterone receptor $(\mathrm{PgR} ; P<0.01)$ were inversely correlated. In multivariate analysis, only high p53 and low bcl2 were independently associated with CAIX positivity. CAIX immunostaining was significantly associated with poor outcome for DFS $(P<0.002)$ and overall survival $(P=0.001)$. In multivariate analysis, a significant interaction was found between CAIX and markers of hormone sensitivity, bcl2 $(P=0.01)$, ER $(P=0.02), \mathrm{PgR}$ $(P=0.02)$, and lymph node involvement $(P=0.04)$, in predicting DFS. Presently, there are few clinical markers of resistance to tamoxifen treatment in ER-positive tumors. CAIX expression in breast cancer patients shows a negative predictive role of treatment efficacy in ER-positive patients on the adjuvant tamoxifen after primary chemo-endocrine therapy. Studies investigating the effects of $\mathrm{pH}$ on tamoxifen uptake and the effects of therapy with $\mathrm{CA}$ inhibitors are planned.
\end{abstract}

Endocrine-Related Cancer (2006) 13 921-930

\section{Introduction}

Hypoxia is a common feature in solid tumors. It is a patho-physiologic consequence of a structurally and functionally disturbed microcirculation and the deterioration of oxygen diffusion conditions (Hockel \& Vaupel 2001). Tumor hypoxia has long been known to be associated with poor survival in cancer patients, since it may contribute to the development of more malignant tumor phenotypes and increase tumor invasiveness and metastatic potential (Harris 2002). Hypoxia also has an important role in the development of resistance to chemotherapy and radiotherapy (Hockel et al. 1996). 
It is recognized that tumor cells under hypoxic conditions maintain a low extracellular $\mathrm{pH}(\mathrm{pHe})$ and a high intracellular pH (Svastova et al. 2004). This confers a survival advantage by possible prevention of tumor cell apoptosis and facilitates the local invasiveness of the tumor by breakdown of the extracellular matrix (Svastova et al. 2004). In addition, the acidic tumor microenvironment reduces uptake of drugs such as anthracyclines, which are weak bases and hence ionized at acid $\mathrm{pH}$ (Gerweck \& Seetharaman 1996, Raghunand et al. 1999, Raghunand \& Gillies 2001).

The carbonic anhydrases (CAs) are zinc metalloenzymes that catalyze the reversible conversion of carbon dioxide to carbonic acid. They participate in $\mathrm{pH}$ regulation, $\mathrm{CO}_{2}$ and $\mathrm{HCO}_{3}$ transport, and water and electrolyte balance (Christianson \& Cox 1999, Supuran et al. 2004).

CA IX is one of the genes associated with tumor cell hypoxia, coding for a transmembrane glycoprotein with an extracellular catalytic domain and an $\mathrm{N}$-terminal proteoglycan domain and regulated by hypoxia inducible factor-1 (HIF-1) (Wykoff et al. 2000). Carbonic anhydrase IX (CAIX) may represent a mechanism of maintaining a $\mathrm{pH}$ difference across the cell membrane. It has been shown that CAIX can acidify the pHe of tumor cells in a culture medium and downregulation reduces the survival of breast tumor cells under hypoxic conditions (Potter \& Harris 2003). Expression of CAIX is localized to the perinecrotic area of tumors and has been observed to start at a median distance of $80 \mu \mathrm{m}$ from a blood vessel, where the oxygen tension drops to $1 \%$ (Beasley et al. 2001). Measurements of tumor hypoxia by Eppendorf microelectrode in advanced cervical cancer have confirmed that there is a direct correlation between hypoxic status and the expression of CAIX (Loncaster et al. 2001). Thus, CAIX is a marker for detecting hypoxia in tumor samples immunohistochemically.

In three studies, the expression of CAIX was associated with poor prognosis independent of the other commonly recognized prognostic parameters such as tumor $(\mathrm{T})$ status, node $(\mathrm{N})$ status, tumor grade, estrogen receptor (ER), and c-erbB2 expression in breast cancer patients (Chia et al. 2001, Bartosova et al. 2002, Span et al. 2003). All these studies involved heterogeneous patient populations submitted postoperatively to different treatment strategies (radiotherapy, chemotherapy, and endocrine therapy) or no therapy.

Primary chemotherapy administered to the breast cancer patients is a useful model to identify baseline features able to predict which patients are most likely to benefit from the cytotoxic treatment and is a way to study new biological markers in relation to the predictive information they provide. In addition, tumor biopsy specimens obtained in matched pair cases at diagnosis and definitive surgery provide valuable information on the interaction between biological markers and treatment.

In the present study, CAIX expression was evaluated in a series of breast cancer specimens obtained before and after primary anthracycline and tamoxifen therapy from breast cancer patients enrolled in a randomized trial of primary therapy, with the aim to: (1) assess the relationship between CAIX expression and diseasefree survival (DFS), (2) evaluate expression in relation to response to the therapy with a single cytotoxic agent, where acid $\mathrm{pH}$ could contribute to drug resistance, and (3) assess changes in CAIX expression after treatment and their relation to the outcome.

\section{Patients and methods}

\section{Patients}

Patients with T2-4 N0-1 breast cancer were recruited in a randomized trial comparing single agent epirubicin (EPI arm) versus epirubicin + tamoxifen (EPI-Tam arm) as primary systemic treatment. The results of this trial have recently been published (Bottini et al. 2005). Two hundred and eleven patients were enrolled in which one hundred and five were randomized to receive epirubicin alone and one hundred and six were randomized to receive epirubicin + tamoxifen. In first presentation, an incision biopsy was performed on each patient and a small tissue sample $(0.5-0.8 \mathrm{~cm})$ was removed. Chemotherapy was started within 2 days of diagnosis. Patients in the EPI arm received $60 \mathrm{mg} / \mathrm{m}^{2}$ epirubicin (Farmorubicina, Pharmacia) by slow i.v. push on days 1 and 2, while patients in the EPI-TAM arm received $60 \mathrm{mg} / \mathrm{m}^{2}$ epirubicin by slow i.v. push on days 1 and 2, and $30 \mathrm{mg}$ tamoxifen (Kessar, Pharmacia) daily. Epirubicin injections were repeated every 21 days for four cycles before definitive surgery, whereas tamoxifen was given continuously until definitive surgery. Surgery was planned after complete clinical reassessment. Quadrantectomy or modified radical mastectomy was performed when indicated in association with complete axillary node dissection. All patients subjected to quadrantectomy underwent irradiation of the residual breast ( 60 Gy delivered over 6 weeks). All patients received four cycles of cyclophosphamide $\left(600 \mathrm{mg} / \mathrm{m}^{2}\right)$, methotrexate $\left(40 \mathrm{mg} / \mathrm{m}^{2}\right)$, and 5 -fluorouracil $\left(600 \mathrm{mg} / \mathrm{m}^{2}\right)$ intravenously on days 1 and 8 every 28 days (CMF regimen) (Zambetti et al. 1997) postoperatively. Patients with ER-positive primary tumor in both arms received tamoxifen $(20 \mathrm{mg}$, 
i.e. lower than the primary dose) starting after surgery, up to progression or for a maximum of 5 years.

\section{Treatment evaluation}

Each month, the size of the primary tumor and axillary lymph nodes, when appreciable, were measured with a caliper by the same clinician. Response was assessed by the clinical measurement of the changes in the product of the two largest diameters recorded in two successive evaluations. According to the World Health Organization criteria, tumor progression (PD) was defined as an increase of at least $25 \%$ in tumor size; stable disease as an increase of less than $25 \%$, or a reduction of less than 50\%; partial response (PR) as a tumor shrinkage greater than 50\%; and complete response (CR) as the complete disappearance of all clinical signs of the disease.

Pathological CR (pCR) was defined as the absence of neoplastic cells in the breast and in the axillary lymph nodes.

\section{Histopathologic grade and immunohistochemistry}

Tumor grade was evaluated using the Nottingham system (Elston \& Ellis 1991). Immunohistochemical evaluation was performed on paraffin-embedded tumor samples obtained at diagnosis and at definitive surgery. bcl2, p53, ER, progesterone receptor (PgR), and Ki67 staining were performed at the Pathology Unit of the Azienda Ospedaliera Istituti Ospitalieri of Cremona (Italy), CAIX staining was assessed at the John Radcliffe Hospital in Oxford, UK.

The immunohistochemical methodology used in Cremona for routine markers is completely described elsewhere (Bottini et al. 2000). Briefly, an antigen retrieval step was performed by heating a tissue section in a citrate buffer. The primary antibodies applied were: ER (mouse monoclonal 6F11 (Novocastra Lab., UK), dilution 1:50, $1 \mathrm{~h}$ incubation at room temperature), $\mathrm{PgR}$ (mouse monoclonal 1A6 (Novocastra Lab.), dilution 1:20, $1 \mathrm{~h}$ incubation at room temperature), Ki67 (mouse monoclonal Mib-1 (Dako, Glostrup, Denmark), dilution 1:30, $1 \mathrm{~h}$ incubation at room temperature), p53 (mouse monoclonal D07 (Novocastra Lab.), dilution 1:100, $1 \mathrm{~h}$ incubation at room temperature), bcl2 (mouse monoclonal 124 (Dako), dilution 1:40, overnight incubation at $4{ }^{\circ} \mathrm{C}$ ), and c-erbB2 (mouse monoclonal CB11 (Novocastra Lab.), overnight incubation at $4{ }^{\circ} \mathrm{C}$ ).

Biotinylated horse anti-mouse $\operatorname{IgG}$ and avidinbiotin-peroxidase complex were applied as a staining method (Vectastatin ABC kit; Vector Laboratories, Inc., Burlingame, CA, USA). A solution containing hydrogen peroxide $(0.06 \% \mathrm{v} / \mathrm{v})$ and diamino-benzidine-4 $\mathrm{HCl}(\mathrm{DAB} ; 0.05 \mathrm{v} / \mathrm{v})$ was used as chromogen.

All samples had a negative control slide (no primary antibody) of an adjacent section to assess the degree of non-specific staining. Positive controls included breast carcinomas known to exhibit high levels of each marker.

All staining was scored by counting the number of positive-stained cells and was expressed as a percentage of the total tumor cells (at least 1000) counted across several representative fields of the section using a standard light microscope equipped with a $10 \times 10$ square graticule. Reproducibility of counting was assessed by a second investigator re-scoring ten slides.

The relative intensity of ER and PgR staining was assessed in a semi-quantitative fashion as previously described by McCarty et al. (1985), incorporating both the intensity and distribution of specific staining. A value (HSCORE) was derived from the sum of the percentages of positive-stained epithelial cells multiplied by the weighted intensity of staining. Specimens were deemed receptor positive if the HSCORE was greater than 100 .

Immunohistochemistry for CAIX was performed on tissues retrieved from the histopathology archives at the Pathology Unit of Cremona, Italy. Two core tissue biopsies, $0.6 \mathrm{~mm}$ in diameter, were taken from selected morphologically representative regions of each paraffin-embedded breast tumor and sections of $5 \mu \mathrm{m}$ thickness of each tissue array block were transferred to the glass slides. Quality control was assessed on each block by haepatoxylin and eosin (H\&E) staining.

Immunohistochemical staining for the endogenous hypoxia marker CAIX was performed with the murine monoclonal antibody M75 (a kind gift from $S$ Pastorekova) at a dilution of 1:50 for $30 \mathrm{~min}$ (Pastorekova et al. 1992). Secondary antibody, an anti-rabbit anti-mouse antibody complex from the Envision HRP kit (Dako) was allowed to incubate for 30 min. Slides were then stained with DAB and counterstained with hematoxylin and mounted. The immunostaining was quantified in carcinoma cells by semi-quantitative scoring as previously described (Colpaert et al. 2003). In brief, a score of 0-2 for the intensity of staining was given ( 0 , no staining; 1 , weak staining; 2, moderate to strong staining). For all comparisons with survival and response, any staining was counted as positive.

The pathologists performing the immunohistochemical evaluations both in the Pathology Unit of Cremona, Italy and John Radcliffe Hospital in Oxford, UK worked in blinded conditions, that is, they did not know the patient outcome and whether the samples 
they examined were obtained from incisional biopsy or definitive surgery.

$\chi^{2}$-test for trend and Fisher's exact test were used when indicated to perform comparisons of proportions. Kruskal-Wallis ANOVA was performed to compare continuous variables. Multivariate logistic regression was used to identify covariates independently associated with CAIX expression. In this analysis, CAIX expression was dichotomized in two classes, ' 0 ' (no immunostaining) and ' 1 ' (presence of immunostaining). DFS was calculated from randomization to the occurrence of disease relapse or death, whichever was first. Overall survival (OS) was calculated from randomization to the date of death. The last follow-up date was considered for DFS and OS (censored), if patients were free from recurrence and alive respectively. The DFS and OS curves were estimated using the Kaplan-Meier method. Unadjusted differences in these estimates were assessed with the log rank test. The Cox proportional hazard model was used to assess the independent predictive role of the clinical-pathological factors in multivariate analysis and the treatment undergone for disease recurrence. The analysis was conducted in two steps: initially, CAIX expression and all classical clinical and pathological factors were included in the multivariate model. Variables not significantly associated with the outcome were then removed from the model in a stepwise backward procedure based on the likelihood ratio $(P<0.10)$. In the second step, the presence of interactions between CAIX and each of the clinical and pathological factors and the treatment undergone was investigated by introducing the appropriate terms describing the interaction between the CAIX and the covariates of interest, one at a time, in the final model obtained in the preceding step. Due to the small sample size and the number of interactions that were evaluated, these analyses must be considered as exploratory, and no attempt was made to build a complete model, where all the significant interaction terms and the associated main effects were included and evaluated simultaneously. All variables included in multivariate analyses were dichotomized and coded ' 0 ' in negative cases and ' 1 ' in positive cases with the exception of Ki67. This latter variable had a left skewed distribution and was modeled using the log transformation. The assumption of proportional hazards was verified by visual inspection of the plots of the log-estimated cumulative hazards in the various strata defined by the covariates, and no major violation

\section{Statistical methodology}

of the assumption was observed. All $P$ values reported were two sided; values less than 0.05 were considered statistically significant.

Statistical analyses were performed using the Statistica for Windows (Tulsa, OK, USA) and SPSS for Windows software packages.

\section{Results}

Out of 211, 183 patients prospectively enrolled in the trial $(86.7 \%)$ had CAIX assessed. For the remaining 28 patients, the blocks had been discarded due to insufficient material. Characteristics of the 183 patients included in this study are shown in Table 1.

Table 1 Patient characteristics

\begin{tabular}{|c|c|c|}
\hline \multicolumn{2}{|l|}{ No. of randomised } & 183 \\
\hline \multicolumn{2}{|l|}{ EPI } & 89 \\
\hline \multicolumn{2}{|l|}{ EPI-TAM } & 94 \\
\hline \multicolumn{2}{|l|}{ Premenopause } & $61(33.9 \%)$ \\
\hline \multicolumn{2}{|l|}{ Postmenopause } & 119 (66.1\%) \\
\hline \multicolumn{3}{|l|}{ Grading } \\
\hline \multicolumn{2}{|l|}{1} & 0 \\
\hline \multicolumn{2}{|l|}{2} & $47(26.5 \%)$ \\
\hline \multicolumn{2}{|l|}{3} & $131(73.5 \%)$ \\
\hline \multicolumn{2}{|l|}{ Missing } & 5 \\
\hline \multicolumn{3}{|l|}{ T stage } \\
\hline \multicolumn{2}{|l|}{$\mathrm{T} 2$} & $138(75.4 \%)$ \\
\hline \multicolumn{2}{|l|}{ T3 } & $26(14.2 \%)$ \\
\hline \multicolumn{2}{|l|}{$\mathrm{T} 4$} & $19(10.4 \%)$ \\
\hline \multicolumn{3}{|l|}{$\mathrm{N}$ status } \\
\hline \multicolumn{2}{|l|}{ No } & $104(56.8 \%)$ \\
\hline \multicolumn{2}{|l|}{ N1 } & 79 (43.2\%) \\
\hline \multicolumn{2}{|l|}{$\mathrm{ER}-$} & $38(20.7 \%)$ \\
\hline \multicolumn{2}{|l|}{$\mathrm{ER}+$} & $145(79.2 \%)$ \\
\hline \multicolumn{2}{|l|}{$\mathrm{PgR}-$} & $92(50.3 \%)$ \\
\hline \multicolumn{2}{|l|}{$\mathrm{PgR}+$} & $91(49.7 \%)$ \\
\hline \multicolumn{2}{|l|}{ p53- } & $91(50.0 \%)$ \\
\hline \multicolumn{2}{|l|}{ p53+ } & 91 (50.0\%) \\
\hline \multicolumn{2}{|l|}{ Missing } & 1 \\
\hline \multicolumn{2}{|l|}{ c-erbB2- } & $134(73.2 \%)$ \\
\hline \multicolumn{2}{|l|}{ c-erbB2+ } & 49 (26.8\%) \\
\hline bcl2- & & 50 (27.5\%) \\
\hline bcl2+ & & $132(72.5 \%)$ \\
\hline Missing & & 1 \\
\hline Response to treat & & \\
\hline Complete respo & & $32(17.6 \%)$ \\
\hline Partial response & & $108(59.3 \%)$ \\
\hline No response & & $42(23.1 \%)$ \\
\hline Not evaluable & & 1 \\
\hline Pathological con & & $6(3.3 \%)$ \\
\hline Baseline & & \\
\hline CAIX $(n=169)$ & 0 & $128(75.7 \%)$ \\
\hline & 1 & $20(11.8 \%)$ \\
\hline & 2 & 21 (12.5\%) \\
\hline After treatment & & \\
\hline CAIX $(n=140)$ & 0 & $110(78.6 \%)$ \\
\hline & 1 & 17 (12.1\%) \\
\hline & 2 & $13(9.3 \%)$ \\
\hline
\end{tabular}


Eighty-nine patients were randomized in the EPI arm, ninety-four were randomized in the EPI-TAM arm. One hundred and sixty-nine patients had CAIX evaluated at the baseline, one hundred and forty had CAIX assessed in residual tumor, one hundred and twenty-six had CAIX assessed both before and after treatment, while fourteen patients had CAIX assessed in the residual tumor histology only. CAIX immunostaining was detected in 41/169 tumor samples collected before treatment (24.2\%) and in 30/140 tumor samples collected afterwards $(21.4 \%)$.

\section{Relationship between CAIX expression and clinical and immunohistochemical prognostic parameters}

As shown in Table 2, CAIX expression at baseline conditions did not correlate with $\mathrm{T}$ status and $\mathrm{N}$ status in univariate analysis. An association of borderline significance (0.08) between CAIX intensity and grade was observed. CAIX expression was directly associated with p53, c-erbB2, and Ki67 expression, while it was inversely associated with bcl2 and steroid hormone receptor status.
In multivariate analysis, however, the only two variables independently associated with CAIX positivity were bcl 2 expression, (odds ratio (OR) $0.2 ; 95 \%$ confidence interval (CI): $0.1-0.5, P=0.0001)$, and p53 expression (OR 2; 95\% CI: 0.9-4.8, $P=0.05$ ).

\section{Effect of treatment on CAIX immunostaining}

In 126 patients with CAIX assessed in matched samples before and after treatments, CAIX positivity was found in 32 baseline tumor samples $(25.4 \%)$ and 27 residual tumor samples to chemotherapy $(21.4 \%)$ respectively. CAIX-positive tumors $(21 / 32)$ at the baseline showed reduction in marker expression (Table 3) and 18 of these became negative at the end of treatment, while CAIX-negative tumors (13/94) at the baseline became positive at the end of treatment. CAIX variation did not differ according to the treatment received (EPI or EPI-Tam; data not shown).

\section{CAIX expression and response to treatment}

Among 169 patients with CAIX assessed at baseline, one patient refused to continue the treatment after the

Table 2 Relationship between baseline CAIX expression and clinical and immunohistochemical parameters

\begin{tabular}{|c|c|c|c|c|}
\hline \multirow[b]{2}{*}{ CAIX intensity } & \multicolumn{3}{|c|}{ Univariate analysis } & \multirow[b]{2}{*}{$P$} \\
\hline & 0 & 1 & 2 & \\
\hline \multicolumn{5}{|l|}{ Grading } \\
\hline 2 & $36 / 125(28.8 \%)$ & $7 / 20(35.0 \%)$ & $1 / 19(5.3 \%)$ & \\
\hline 3 & $89 / 125(71.2 \%)$ & $13 / 20(65.0 \%)$ & $18 / 19(94.7 \%)$ & $0.08^{*}$ \\
\hline p53 & $56 / 128(43.7 \%)$ & $14 / 20(70.0 \%)$ & $14 / 20(70.0 \%)$ & $0.007^{*}$ \\
\hline c-erbB2 & $48 / 128(37.5 \%)$ & $13 / 20(65.0 \%)$ & $13 / 21(61.9 \%)$ & $<0.01^{*}$ \\
\hline bcl2 & $105 / 127$ (82.6\%) & $14 / 20(70.0 \%)$ & $6 / 21(28.6 \%)$ & $0.000^{*}$ \\
\hline ER & $111 / 128(86.7 \%)$ & $15 / 20(75.0 \%)$ & $11 / 21(52.4 \%)$ & $0.000^{*}$ \\
\hline $\mathrm{PgR}$ & $71 / 128$ (55.5\%) & $7 / 20(35.0 \%)$ & $6 / 21(28.6 \%)$ & $<0.01^{*}$ \\
\hline Ki67 mean & 19.8 & 26.0 & 35.8 & 0.02 \\
\hline$(95 \% \mathrm{Cl})$ & $(17.1-22.5)$ & $(13.6-38.5)$ & $(23.2-48.5)$ & \\
\hline T2 & $98 / 128(76.5 \%)$ & $13 / 20(65.0 \%)$ & $16 / 21(76.2 \%)$ & \\
\hline T3-4 & $30 / 128(23.5 \%)$ & $7 / 20(35.0 \%)$ & $5 / 21(23.8 \%)$ & 0.68 \\
\hline \multirow[t]{3}{*}{$\mathrm{N}+$} & $53 / 128(41.4 \%)$ & $10 / 20(50.0 \%)$ & $11 / 21(52.4 \%)$ & $0.12^{*}$ \\
\hline & \multicolumn{3}{|c|}{ Multivariate analysis $^{\dagger}$} & \\
\hline & Odds ratio & $95 \%$ confi & vals & $P$ \\
\hline \multicolumn{5}{|c|}{ Variables in the model } \\
\hline p53 & 2 & & & 0.05 \\
\hline bcl2 & 0.2 & & & 0.0001 \\
\hline \multicolumn{5}{|c|}{ Variables failing to enter the model } \\
\hline ER & 0.9 & & & 0.6 \\
\hline Tumor grade & 0.9 & & & 0.9 \\
\hline $\mathrm{PgR}$ & 0.6 & & & 0.2 \\
\hline Log Ki67 & 1 & & & 0.7 \\
\hline c-erbB2 & 1.7 & & & 0.17 \\
\hline
\end{tabular}

* $\chi 2$ for trend; 'CAIX was dicotomized and coded as '0' (no expression) and ' 1 ' (expression). 
Table 3 CAIX individual changes and CAIX expression before and after treatment

\begin{tabular}{lcccc}
\hline & Overall & $\begin{array}{c}\text { No } \\
\text { variation }\end{array}$ & Increase & Decrease \\
\hline CAIX +ve & 32 & $11(34.4 \%)$ & n.a. & $21(65.6 \%)$ \\
CAIX - ve & 94 & $81(86.2 . \%)$ & $13(13.8 \%)$ & n.a. \\
\hline
\end{tabular}

first cycle and was not assessable for response; $129(76.7 \%)$ out of 168 assessable cases attained a clinical response (complete + partial), 30 (17.8\%) cases showed a CR and $99(58.9 \%)$ had a PR. At postchemotherapy residual histology, six patients (3.6\%) had a pCR. As previously published, there was no significant difference in treatment responses between the epirubicin and epirubicin + tamoxifen arms (76 vs $82 \%$ respectively) (Bottini et al. 2005). According to the CAIX status, overall response was observed in 100 out of 127 patients $(78.7 \%)$ with CAIX-negative tumors and 29 out of 41 patients (70.7\%) with CAIX-positive tumors, and CR was observed in 25/127 (19.7\%) CAIX-negative and in 5/41 (12.2\%) CAIX-positive patients respectively. None of these differences was statistically significant. All the six pCRs were confined to patients with CAIX negative primary tumor $(P=0.33$, Fisher's exact test).

\section{CAIX expression and disease outcome}

Out of 183, 45 patients relapsed (24.6\%) and 21 $(11.5 \%)$ died of disease. As shown in Fig. 1a and b, CAIX expression was significantly associated with poor outcome both in terms of DFS and OS.

In univariate analysis, tumor grade $(P=0.04), \mathrm{T}$ $(P=0.0001)$ and $\mathrm{N}$ status $(P=0.000)$, ER $(P=0.003)$, $\operatorname{PgR}(P=0.03)$, and bcl-2 expression $(P=0.002)$, in addition to CAIX expression, were significantly associated with disease recurrence, while the correlation with disease recurrence of $\operatorname{Ki67}(P=0.10)$, p53 $(P=0.06)$, and c-erbB2 expression $(P=0.07)$ just failed to attain statistical significance. Menopausal status was not associated with disease recurrence $(P=0.29)$.

In multivariate analysis, CAIX expression was not an independent predictor of DFS (Table 4). Tumor size (hazard ratio (HR) 2.7; 95\% CI: 1.4-5.4, $P=0.003$ ), lymph node involvement (HR 2.8; 95\% CI: 1.4-5.7, $P=0.004$ ), and bcl 2 status (HR $0.4 ; 95 \%$ CI: $0.2-0.8$, $P=0.008)$ were retained in the final model. Treatmentinduced changes in CAIX were not correlated with DFS in univariate analysis (data not shown).
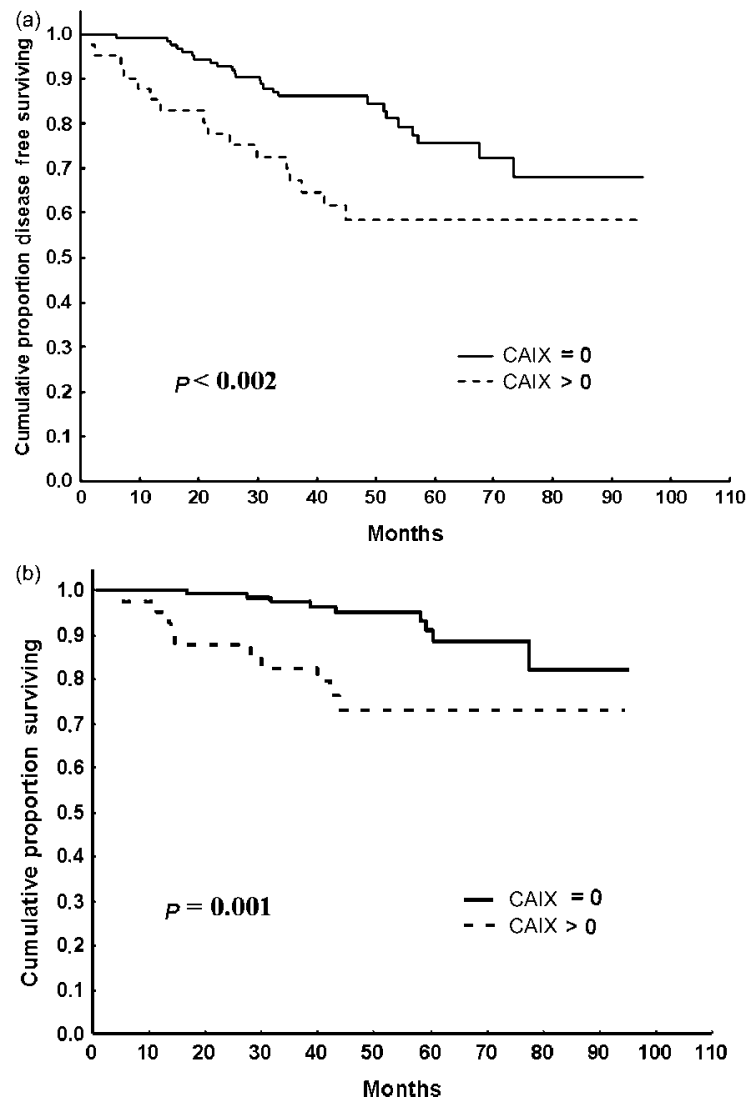

Figure 1 (a) Disease-free and (b) overall survival according to CAIX status.

\section{Effect of CAIX on DFS in different subgroups according to clinical and pathological parameters}

In the exploratory analyses aimed at evaluating the interactions on DFS between CAIX and each of the clinical and pathological parameters, a significant positive interaction was found between CAIX and

Table 4 Multivariate Cox analysis for independent factors predictive for disease recurrence

\begin{tabular}{|c|c|c|c|}
\hline & $\begin{array}{l}\text { Hazard } \\
\text { ratio }\end{array}$ & $\begin{array}{l}\text { 95\% confidence } \\
\text { intervals }\end{array}$ & $\boldsymbol{P}$ \\
\hline \multicolumn{4}{|c|}{ Variables in the final model } \\
\hline Nodal status & 3.0 & $1.5-6.0$ & 0.002 \\
\hline $\mathrm{T}$ & 2.6 & $1.3-5.0$ & 0.007 \\
\hline bcl2 & 0.4 & $0.2-0.8$ & 0.008 \\
\hline \multicolumn{4}{|c|}{ Variables failing to enter the model } \\
\hline c-erbB2 & 0.7 & $0.3-1.5$ & 0.4 \\
\hline $\mathrm{PgR}$ & 1.1 & $0.5-2.6$ & 0.8 \\
\hline ER & 0.7 & $0.3-1.9$ & 0.5 \\
\hline Grading & 1.4 & $0.5-3.8$ & 0.4 \\
\hline Log Ki67 & 2.5 & $0.8-8.0$ & 0.1 \\
\hline CAIX & 1.6 & $0.8-3.2$ & 0.2 \\
\hline p53 & 1.2 & $0.6-2.4$ & 0.5 \\
\hline
\end{tabular}


Table 5 Subgroup analysis of disease-free survival of CAIXpositive patients versus CAIX negative ones within strata formed by each prognostic factor

\begin{tabular}{lccc}
\hline & Hazard ratio* & $\begin{array}{c}95 \% \text { confidence } \\
\text { interval }\end{array}$ & $\boldsymbol{P}$ \\
\hline $\begin{array}{l}\text { Estrogen receptor } \\
\text { Positive }\end{array}$ & 3.2 & $1.4-7.4$ & $0.02^{\dagger}$ \\
$\quad$ Negative & 0.7 & $0.2-3.1$ & \\
$\begin{array}{l}\text { Progesterone receptor } \\
\text { Positive }\end{array}$ & 17.1 & $3.2-90.5$ & $0.02^{\dagger}$ \\
$\quad$ Negative & 1.0 & $0.4-2.6$ & \\
bcl2 & & & \\
$\quad$ Positive & 2.8 & $1.1-7.1$ & $0.01^{\dagger}$ \\
$\quad$ Negative & 0.6 & $0.2-1.9$ & \\
Node status & & $1.0-4.8$ & $0.04^{\dagger}$ \\
$\quad$ Positive & 2.2 & $0.2-4.4$ & \\
Negative & 1.1 & & \\
\hline
\end{tabular}

*From a Cox model in which all variables were included as covariates. ${ }^{\dagger}$ Test for interaction. The interaction terms were, one at a time, added to the final model. Before testing another interaction, both the covariate and the interaction term were removed from the model. Terms for interaction of CAIX with p53, tumor grade, log Ki67, c-erbB2, and T status were not significantly associated with outcome and were not included in the table.

bcl2 $(P=0.01)$, ER $(P=0.02), \operatorname{PgR}(P=0.02)$, and lymph node involvement $(P=0.04)$. The interaction between CAIX and the various factors in predicting DFS is depicted in Table 5. As shown, CAIX positivity was clearly associated with a two- to threefold increased risk of relapse in patients with tumors positive for markers of responsiveness to endocrine therapy, such as ER, PgR and bcl2, and in nodepositive patients, whereas no association was seen in patients negative for these markers and in nodenegative patients (Table 5). No variation in the prognostic effect of CAIX was seen across subgroups

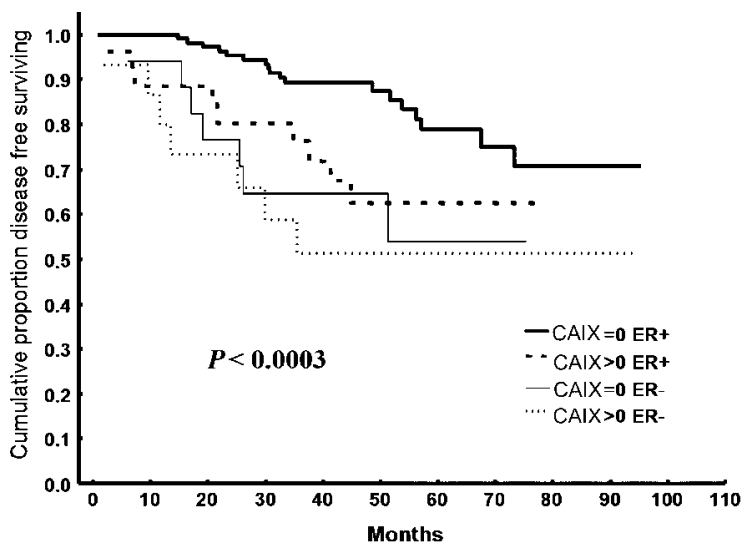

Figure 2 Disease-free survival of patient subgroups stratified according to CAIX and estrogen receptor (ER) status. of the other factors. The interaction between CAIX and ER status is shown in Fig. 2 in which the predictive role of CAIX for DFS in univariate analysis is confined to ER-positive patients.

\section{Discussion}

Hypoxia has been implicated as an important component in tumor progression and spread. We initially identified CAIX to be hypoxia inducible in several epithelial cell lines and its expression was closely correlated with the presence of necrosis, believed to be an indicator of local hypoxia, within invasive breast tumors (Leek et al. 1999). As a measure of tumor cell hypoxia CAIX was shown to be a prognostic factor in different cancers (Koukourakis et al. 2001, Loncaster et al. 2001, Hui et al. 2002, Swinson et al. 2003, Bui et al. 2004, Hussain et al. 2004). These studies have also revealed that the expression of this biomarker is restricted to the transformed, dysplastic, and malignant epithelial cells and is rarely expressed in benign or normal tissue.

However, in the studies published to date, CAIX was assessed in tumor samples of the patients submitted to heterogeneous adjuvant treatments or no treatment at all after surgery. From these studies, it is very difficult to draw conclusions on the predictive role of this marker on treatment efficacy and the heterogeneous treatments administered could have lead to biased results with respect to the disease outcome, while predictive factors can be best evaluated in the context of a prospective randomized clinical trial $(31,32)$. In the present study, CAIX evaluation was performed in a series of patients prospectively enrolled in a single institution phase III trial comparing epirubicin versus epirubicin + tamoxifen, as primary systemic treatment (Bottini et al. 2005). In addition, after surgery, all patients received i.v. CMF regimen in adjuvant setting and those patients with ER-positive primary tumor in both treatment arms underwent 5 year adjuvant tamoxifen therapy. As previously observed, the CAIX expression was not related to disease stage (Chia et al. 2001), whereas it was significantly associated with biological tumor prognostic features, such as p53, Ki67, c-erbB2 expression as well as negative steroid hormone receptor status, and negative bcl 2 status (Leek et al. 1999). Bertosova et al. (2002) reported a weak, but significant, correlation of CAIX in breast cancer with c-erbB2 expression. We confirmed this association suggesting that CAIX with its reversed correlation with ER status and positive correlation with c-erbB2 is associated with resistance to endocrine therapy. 
This study, however, is the first to show that CAIX expression is related to the expression of genes regulating apoptosis and proliferative activity. As previously reported, all the biological variables considered in this study are reciprocally correlated (Bottini et al. 2000). When the variables associated with CAIX expression in univariate analysis were included in a multivariate logistic regression model, only p53 and bcl 2 showed an independent relationship with CAIX expression. The relationship reflects the inverse association with ER, since bcl2 is an estrogen-regulated gene and p53 expression is associated with ER-negative cases.

Tumor hypoxia regulates many changes in gene expression. In particular, hypoxia selects for p53-mutations (Schmid et al. 2004) (most p53 detected by immunohistochemical procedures is usually the mutated form) leading to apoptosis inhibition. The direct relationship between CAIX and p53 supports these data, and in cell lines, it has been shown that wildtype p53 suppresses CAIX expression (Kaluzova et al. 2004), thus providing a mechanism for co-expression in some cases, although most CAIX-positive cases were p53 negative. The inverse relationship with bcl 2 reflects the regulation of bcl2 by ER, with hormone receptors being reciprocally related to CAIX.

In this study, CAIX was not predictive of clinical response overall, although no patient with a CAIXpositive tumor had a pCR. We gave patients the maximal dose of epirubicin that can be given without marrow support or cardioprotection on a three weekly basis, as a single agent. The hypothesis tested was that CAIX could mediate one mechanism of resistance relating to poor drug uptake. There was no difference in response in those treated by epirubicin alone versus tamoxifen. Although an effect of CAIX expression was not detected on response, this may be difficult because of the large tumor mass, and effects on elimination of micrometastases could be a more relevant endpoint and represented by poorer DFS.

Few tumors changed the CAIX status before and after treatment. The number of patients with a CAIXnegative tumor at baseline, which becomes positive afterwards is similar to that of patients showing an opposite pattern. The percentage of patients with CAIXpositive tumors did not differ significantly before and after treatment. These data suggest that, as a whole, CAIX immunostaining is not influenced by treatment.

In this series, CAIX expression was significantly associated with poor DFS and OS, confirming previous observations in breast cancer (Chia et al. 2001, Colpaert et al. 2003, Span et al. 2003). However, in multivariate analysis, CAIX failed to be an independent predictor of DFS when adjusted for $\mathrm{T}$ status, $\mathrm{N}$ status, p53, bcl2, Ki67, steroid hormone receptors, and treatment. This observation is in contrast with previous publications (Chia et al. 2001, Span et al. 2003, Chia \& Yorida 2004). It should be noted, however, that the HR of 1.6 obtained with CAIX in the present study is superimposable to HRs of CAIX reported in multivariate analyses performed in previous studies (Chia et al. 2001, Chia \& Yorida 2004, Yorida et al. 2004). These data provide further information in favor of the negative prognostic significance of CAIX expression.

We observed a statistical interaction of CAIX with $\mathrm{ER}, \mathrm{PgR}$, and bcl2 in determining DFS, indicating that CAIX prognostic significance is limited to (or more pronounced in) patients with hormone-responsive tumors. Hypoxia can cause endocrine therapy resistance as has been shown recently in cell lines in the tissue culture (Cooper et al. 2004, Coradini et al. 2004), thereby contributing to increased drug resistance. Tamoxifen in particular is a weak basic drug $\left(\mathrm{p} K_{\mathrm{a}}=8.8\right)$, so that possible mechanisms of hypoxiainduced resistance to this drug relate to the acid outside $\mathrm{pH}$ gradients that reduce the partitioning of weak basic drugs into the relatively alkaline cells. This phenomenon has its basis in 'ion trapping', wherein weak bases partition into, and are sequestered by acidic compartments, such as the extracellular matrix (Roos 1978). This occurs because uncharged, organic-free bases are more permeable than their protonated and charged counterparts and establish equal concentrations on either side of the cell membrane (Gillies 1978). There is evidence that decreased uptake is a possible mechanism of resistance in hormone-resistant breast cancers on tamoxifen therapy, although how this occurred was not described (Dowsett et al. 1995). The results of the present paper suggest a contribution of CAIX expression in the tamoxifen resistance. Since the patient population with ER-positive tumors was homogeneously submitted to 5 year adjuvant tamoxifen in addition to chemotherapy, it is not possible to assess whether the observed interaction is due to an antagonizing effect of CAIX positivity on the efficacy of tamoxifen or it may be attributable to a true interaction between two different tumor characteristics.

In conclusion, there are presently few clinical markers of resistance to tamoxifen treatment in ERpositive tumors. CAIX expression in breast cancer patients shows a negative predictive role of treatment efficacy in ER-positive patients on the adjuvant tamoxifen after primary chemo-endocrine therapy. Studies investigating the effects of $\mathrm{pH}$ on tamoxifen uptake and the effects of therapy with CA inhibitors are planned. 


\section{Acknowledgements}

This work was supported in part by the 'Associazione Patologia Oncologica Mammaria - APOM', Cremona, Italy, by the Association of 'Amici dell'Ospedale di Cremona', by a grant from the Consiglio Nazionale Ricerche (CNR), Rome, Italy, by Cancer Research UK (CRUK), UK and the European Union via the Euroxy Grant. The authors declare that there is no conflict of interest that would prejudice the impartiality of this scientific work.

\section{References}

Bartosova M, Parkkila S, Pohlodek K, Karttunen TJ, Galbavy S, Mucha V, Harris AL, Pastorek J \& Pastorekova S 2002 Expression of carbonic anhydrase IX in breast is associated with malignant tissues and is related to overexpression of c-erbB2. Journal of Pathology 197 314-321.

Beasley NJ, Wykoff CC, Watson PH, Leek R, Turley H, Gatter K, Pastorek J, Cox GJ, Ratcliffe P \& Harris AL 2001 Carbonic anhydrase IX, an endogenous hypoxia marker, expression in head and neck squamous cell carcinoma and its relationship to hypoxia, necrosis, and microvessel density. Cancer Research 61 5262-5267.

Bottini A, Berruti A, Bersiga A, Brizzi MP, Brunelli A, Gorzegno G, DiMarco B, Aguggini S, Bolsi G, Cirillo F et al. $2000 \mathrm{p} 53$ but not bcl-2 immunostaining is predictive of poor clinical complete response to primary chemotherapy in breast cancer patients. Clinical Cancer Research 6 2751-2758.

Bottini A, Berruti A, Brizzi MP, Bersiga A, Generali D, Allevi G, Aguggini S, Bolsi G, Bonardi S, Tondelli B et al. 2005 Cytotoxic and antiproliferative activity of the single agent epirubicin versus epirubicin plus tamoxifen as primary chemotherapy in human breast cancer: a single-institution phase III trial. Endocrine-Related Cancer 12 383-392.

Bui MH, Visapaa H, Seligson D, Kim H, Han KR, Huang Y, Horvath S, Stanbridge EJ, Palotie A, Figlin RA et al. 2004 Prognostic value of carbonic anhydrase IX and KI67 as predictors of survival for renal clear cell carcinoma. Journal of Urology 171 2461-2466.

Chia SK \& Yorida E 2004 Carbonic anhydrase IX (CaIX) is an independent poor prognostic factor in early stage breast cancer: results from a large population-based tissue microarray (TMA) series. Proceedings of the 27th San Antonio Breast Cancer Symposium. Abstract 3015.

Chia SK, Wykoff CC, Watson PH, Han C, Leek RD, Pastorek J, Gatter KC, Ratcliffe P \& Harris AL 2001 Prognostic significance of a novel hypoxia-regulated marker, carbonic anhydrase IX, in invasive breast carcinoma. Journal of Clinical Oncology 19 3660-3668.

Christianson DW \& Cox JD 1999 Catalysis by metalactivated hydroxide in zinc and manganese metalloenzymes. Annual Reviews in Biochemistry 68 33-57.
Colpaert CG, Vermeulen PB, Fox SB, Harris AL, Dirix LY \& Van Marck EA 2003 The presence of a fibrotic focus in invasive breast carcinoma correlates with the expression of carbonic anhydrase IX and is a marker of hypoxia and poor prognosis. Breast Cancer Research and Treatment 81 137-147.

Cooper C, Liu GY, Niu YL, Santos S, Murphy LC \& Watson PH 2004 Intermittent hypoxia induces proteasome-dependent down-regulation of estrogen receptor alpha in human breast carcinoma. Clinical Cancer Research 10 8720-8727.

Coradini D, Pellizzaro C, Speranza A \& Daidone MG 2004 Hypoxia and estrogen receptor profile influence the responsiveness of human breast cancer cells to estradiol and antiestrogens. Cellular and Molecular Life Sciences 61 76-82.

Dowsett M, Johnston SR, Iveson TJ \& Smith IE 1995 Response to specific anti-oestrogen (ICI182780) in tamoxifen-resistant breast cancer. Lancet 345525.

Elston CW \& Ellis IO 1991 Pathological prognostic factors in breast cancer. I. The value of histological grade in breast cancer: experience from a large study with long-term follow-up. Histopathology 19 403-410.

Gerweck LE \& Seetharaman K 1996 Cellular pH gradient in tumor versus normal tissue: potential exploitation for the treatment of cancer. Cancer Research 56 1194-1198.

Gillies D 1978 Intracellular pH: methods and applications. Current Topics in Bioenergetics 9 63-87.

Harris AL 2002 Hypoxia - a key regulatory factor in tumour growth. Nature Reviews Cancer 2 38-47.

Hockel M \& Vaupel P 2001 Tumor hypoxia: definitions and current clinical, biologic, and molecular aspects. Journal of the National Cancer Institute 93 266-276.

Hockel M, Schlenger K, Aral B, Mitze M, Schaffer U \& Vaupel P 1996 Association between tumor hypoxia and malignant progression in advanced cancer of the uterine cervix. Cancer Research 56 4509-4515.

Hui EP, Chan AT, Pezzella F, Turley H, To KF, Poon TC, Zee B, Mo F, Teo PM, Huang DP et al. 2002 Coexpression of hypoxia-inducible factors 1alpha and 2alpha, carbonic anhydrase IX, and vascular endothelial growth factor in nasopharyngeal carcinoma and relationship to survival. Clinical Cancer Research $\mathbf{8}$ 2595-2604.

Hussain SA, Palmer DH, Ganesan R, Hiller L, Gregory J, Murray PG, Pastorek J, Young L \& James ND 2004 Carbonic anhydrase IX, a marker of hypoxia: correlation with clinical outcome in transitional cell carcinoma of the bladder. Oncology Reports 11 1005-1010.

Kaluzova M, Kaluz S, Lerman MI \& Stanbridge EJ 2004 DNA damage is a prerequisite for p53-mediated proteasomal degradation of HIF-1alpha in hypoxic cells and downregulation of the hypoxia marker carbonic anhydrase IX. Molecular and Cellular Biology 24 5757-5766.

Koukourakis MI, Giatromanolaki A, Sivridis E, Simopoulos K, Pastorek J, Wykoff CC, Gatter KC \& Harris AL 2001 Hypoxia-regulated carbonic anhydrase-9 (CA9) relates to 
poor vascularization and resistance of squamous cell head and neck cancer to chemoradiotherapy. Clinical Cancer Research 7 3399-3403.

Leek RD, Landers RJ, Harris AL \& Lewis CE 1999 Necrosis correlates with high vascular density and focal macrophage infiltration in invasive carcinoma of the breast. British Journal of Cancer 79 991-995.

Loncaster JA, Harris AL, Davidson SE, Logue JP, Hunter RD, Wycoff CC, Pastorek J, Ratcliffe PJ, Stratford IJ \& West CM 2001 Carbonic anhydrase (CA IX) expression, a potential new intrinsic marker of hypoxia: correlations with tumor oxygen measurements and prognosis in locally advanced carcinoma of the cervix. Cancer Research $\mathbf{6 1}$ 6394-6399.

McCarty KS Jr, Miller LS, Cox EB, Konrath J \& McCarty KS, Sr 1985 Estrogen receptor analyses. Correlation of biochemical and immunohistochemical methods using monoclonal antireceptor antibodies. Archives in Pathology and Laboratory Medicine 109 716-721.

Pastorekova S, Zavadova Z, Kostal M, Babusikova O \& Zavada J 1992 A novel quasi-viral agent, MaTu, is a twocomponent system. Virology 187 620-626.

Potter CP \& Harris AL 2003 Diagnostic, prognostic and therapeutic implications of carbonic anhydrases in cancer. British Journal of Cancer 89 2-7.

Raghunand N \& Gillies RJ 2001 pH and chemotherapy. Novartis Foundation Symposium 240 199-211 (discussion 265-268).

Raghunand N, He X, van Sluis R, Mahoney B, Baggett B, Taylor CW, Paine-Murrieta G, Roe D, Bhujwalla ZM \& Gillies RJ 1999 Enhancement of chemotherapy by manipulation of tumour $\mathrm{pH}$. British Journal of Cancer $\mathbf{8 0}$ 1005-1011.
Roos A 1978 Weak acids, weak bases and intracellular pH. Respiratory Physiology 33 27-30.

Schmid T, Zhou J \& Brune B 2004 HIF-1 and p53: communication of transcription factors under hypoxia. Journal of Cellular and Molecular Medicine 8 423-431.

Span PN, Bussink J, Manders P, Beex LV \& Sweep CG 2003 Carbonic anhydrase- 9 expression levels and prognosis in human breast cancer: association with treatment outcome. British Journal of Cancer 89 271-276.

Supuran CT, Vullo D, Manole G, Casini A \& Scozzafava A 2004 Designing of novel carbonic anhydrase inhibitors and activators. Current Medicinal Chemistry Cardiovascular \& Hematological Agents 2 51-70.

Svastova E, Hulikova A, Rafajova M, Zat'ovicova M, Gibadulinova A, Casini A, Cecchi A, Scozzafava A, Supuran CT, Pastorek J et al. 2004 Hypoxia activates the capacity of tumor-associated carbonic anhydrase IX to acidify extracellular pH. FEBS Letters 577 439-445.

Swinson DE, Jones JL, Richardson D, Wykoff C, Turley H, Pastorek J, Taub N, Harris AL \& O’Byrne KJ 2003 Carbonic anhydrase IX expression, a novel surrogate marker of tumor hypoxia, is associated with a poor prognosis in non-small-cell lung cancer. Journal of Clinical Oncology 21 473-482.

Wykoff CC, Beasley NJ, Watson PH, Turner KJ, Pastorek J, Sibtain A, Wilson GD, Turley H, Talks KL, Maxwell PH et al. 2000 Hypoxia-inducible expression of tumorassociated carbonic anhydrases. Cancer Research 60 7075-7083.

Zambetti M, Giacobone A, Terenziani M, Zucchinelli P, Demicheli R, Biasi S, Piotti P, Bartoli C, Valagussa P \& Bonadonna G 1997 Sequential adriamycin and CMF in metastatic breast cancer. Oncologist 2 223-227. 